\title{
Favourable metabolic profile sustains mitophagy and prevents metabolic abnormalities in metabolically healthy obese individuals
}

\author{
Shipra Bhansali ${ }^{1}$, Anil Bhansali ${ }^{2}$ and Veena Dhawan ${ }^{1 *}$ (D)
}

\begin{abstract}
Background: Obesity-mediated oxidative stress results in mitochondrial dysfunction, which has been implicated in the pathogenesis of metabolic syndrome and T2DM. Recently, mitophagy, a cell-reparative process has emerged as a key facet in maintaining the mitochondrial health, which may contribute to contain the metabolic abnormalities in obese individuals. However, the status of mitophagy in metabolically healthy obese (MHO) and metabolically abnormal diabetic obese (MADO) subjects remains to be elucidated. Hence, the present study aims to unravel the alterations in mitochondrial oxidative stress (MOS) and mitophagy in these subjects.
\end{abstract}

Methods: 60 subjects including MHNO (metabolically healthy non-obese), MHO and MADO were enrolled as per the Asian criteria for obesity ( $n=20$ each). Biochemical parameters, MOS indices, transcriptional and translational expression of mitophagy markers (PINK1, PARKIN, MFN2, NIX, LC3-II, and LAMP-2), and transmission electron microscopic (TEM) studies were performed in peripheral blood mononuclear cells.

Results: The $\mathrm{MHO}$ subjects displayed a favorable metabolic profile, despite accompanied by an increased adiposity as compared to the MHNO group; while MADO group exhibited several metabolic abnormalities, inspite of similar body composition as MHO subjects. A progressive rise in the MOS was observed in MHO and MADO subjects as compared to the MHNO group, and it showed a positive and significant correlation with the body composition in these groups. Further, mitophagy remained unaltered in the $\mathrm{MHO}$ group, while it was significantly downregulated in the MADO group. In addition, TEM studies revealed a significant increase in the percentage of damaged mitochondria in MADO patients as compared to other groups, while $\mathrm{MHO}$ and $\mathrm{MHNO}$ groups did not show any significant alterations for the same.

Conclusion: A favorable metabolic profile and moderate levels of MOS in the MHO group may play a crucial role in the sustenance of mitophagy, which may further limit the aggravation of MOS, inflammation, and emergence of metabolic aberrations in contrast to MADO subjects, who exhibited multiple metabolic abnormalities and attenuated mitophagy. Therefore, these $\mathrm{MHO}$ subjects are likely to be at a lower risk of developing metabolic syndrome and T2DM.

Keywords: Metabolically healthy non-obese (MHNO), Metabolically healthy obese (MHO), Metabolically abnormal diabetic obese (MADO), Mitochondrial oxidative stress and mitophagy

\footnotetext{
*Correspondence: veena447@gmail.com

1 Department of Experimental Medicine and Biotechnology, Postgraduate Institute of Medical Education and Research (PGIMER), Research Block-B, Chandigarh 160012, India

Full list of author information is available at the end of the article
} 


\section{Background}

Obesity is associated with numerous metabolic alterations such as insulin resistance, glucose intolerance, and dyslipidemia. The constellation of these risk factors constitute the metabolic syndrome that predisposes the individuals to the risk of developing type 2 diabetes mellitus (T2DM) and cardiovascular diseases [1, 2]. However, a subset of obese subjects do not exhibit an increased risk of developing these aforementioned metabolic abnormalities, and they have been identified as metabolically healthy obese (MHO) subjects, accounting for approximately $20-30 \%$ of the obese adult population [3]. A study by Zheng et al. reported that the prevalence of $\mathrm{MHO}$ subjects was $27.9 \%$ in the obese adult Chinese population [4]. Similarly, another study from Southern India described the prevalence of $\mathrm{MHO}$ as $13.3 \%$ in the adult population [5].

MHO subjects are characterized by the absence of several metabolic abnormalities such as insulin resistance, dyslipidemia, glucose intolerance, hypertension, inspite of increased adiposity, but have a favorable inflammatory profile as compared to the metabolically abnormal diabetic obese (MADO) individuals, who are usually accompanied by severe metabolic abnormalities. However, the underlying mechanisms contributing towards the relative protection against obesity-induced metabolic complications remains largely unknown.

Metabolic syndrome is associated with oxidative stress and mitochondrial dysfunction. Mitochondrion, a vital cell organelle, is the primary site for endogenous ROS generation and glucose metabolism. High calorific diet intake leads to an energetic overload, which in turn results in an ineffective mitochondrial oxidative phosphorylation with an abnormal generation of mitochondrially-derived superoxide anions, eventually damaging the mitochondrial-related DNA and proteins [6]. Moreover, mitochondria are also involved in the insulin biosynthesis and secretion as well as in fuel metabolism at insulin target sites [7]. Recently, a study by Chattopadhyay et al. reported that the mitochondrial reactive oxygen species (ROS) production was higher in the subcutaneous adipose tissue of the obese T2DM subjects as compared to the obese non-diabetic subjects and nonobese T2DM patients, indicating that the mitochondrial dysfunction with the enhanced ROS production may contribute to metabolic abnormalities in obesity and T2DM [8]. Similarly, another study by Befroy et al. reported that the mitochondrial dysfunction is associated with insulin resistance in skeletal muscle of T2DM patients [9].

In order to maintain the mitochondrial health, a process known as mitophagy, and its implications in metabolic syndrome have emerged as a novel area of research.
Mitophagy, also known as mitochondrial autophagy involves the selective sequestration of damaged or defunct mitochondria via autophagic vesicles, followed by their subsequent lysosomal degradation, thereby playing a crucial role in the regulation of mitochondrial quality and quantity control [10]. In this context, Twig et al. reported that mitophagy is important in the turnover of dysfunctional mitochondria in $\beta$-cells, which is required for its optimal functioning, and the dysregulation of this process might predispose to the risk of developing metabolic syndrome and T2DM [11].

Mitophagy is a multi-step, evolutionary conserved process, regulated by various genes including PTEN-induced putative kinase 1 (PINK1), PARKIN, NIP3-like protein $\mathrm{X}(N I X)$ and mitofusin2 (MFN2), involved in the priming of the damaged/defunct mitochondria, followed by their recruitment to the autophagosome machinery via another protein, microtubule-associated protein light chain 3 (LC3), a marker of autophagosomal membrane. The autophagic vesicles carrying the defunct mitochondria, known as mitophagosomes, subsequently fuse with the lysosomes, which is facilitated by lysosome-associated membrane protein-2 (LAMP-2) [12]. Thereafter, the engulfed mitochondrial cargo is degraded by lysosomal hydrolases, hence maintaining the mitochondrial health.

Mounting evidence reveal that the obesity and/or metabolic abnormalities-mediated inflammation, and MOS are closely associated with mitochondrial dysfunction $[13,14]$. Therefore, the regulated elimination of dysfunctional mitochondria is of primary importance to suppress the obesity-induced metabolic disorders. Based on the above evidence, we hypothesized that mitophagy, a cellreparative phenomenon, which is essential for restoring the mitochondrial function, might attribute to the pathophysiology of obesity. Hence, the present study aimed to assess the magnitude of mitochondrial oxidative stress and mitophagy in metabolically healthy non-obese (MHNO), metabolically healthy obese (MHO) and metabolically abnormal diabetic obese (MADO) subjects, who were accompanied with varying degree of adiposity and/ or metabolic abnormalities.

\section{Methods \\ Study population}

Sixty, age, and gender-matched non-obese (BMI $<23 \mathrm{~kg} /$ $\mathrm{m}^{2}$ ) and obese (BMI $\left.\geq 25 \mathrm{~kg} / \mathrm{m}^{2}\right)$ subjects were screened as per the Asian criteria for obesity and enrolled at endocrine out-patient department of the Postgraduate Institute of Medical Education and Research (PGIMER), Chandigarh, India [15]. The revised National Cholesterol Education Programs's Adult treatment Panel III report for Asian population defined the criteria for the metabolic syndrome as follows: WC $>80 \mathrm{~cm}$ in men, $>90$ 
$\mathrm{cm}$ in women, $\mathrm{BP}>130 / 85 \mathrm{mmHg}, \mathrm{FPG} \geq 100 \mathrm{mg} / \mathrm{dl}$ or $\mathrm{HbA}_{1 \mathrm{C}}>5.6 \%, \mathrm{TG} \geq 150 \mathrm{mg} / \mathrm{dl}$ and HDL-C $<40 \mathrm{mg} / \mathrm{dl}$ in men or $<50 \mathrm{mg} / \mathrm{dl}$ in women [16]. According to the above mentioned criteria, the study participants were divided into 3 groups: (a) Metabolically healthy non-obese (MHNO): $\mathrm{BMI}<23 \mathrm{~kg} / \mathrm{m}^{2}$ and $\leq 1$ metabolic risk factor. (b) Metabolically healthy obese (MHO): BMI $\geq 25 \mathrm{~kg} / \mathrm{m}^{2}$ and $\leq 1$ metabolic risk factor. (c) Metabolically abnormal diabetic obese (MADO): $\mathrm{BMI} \geq 25 \mathrm{~kg} / \mathrm{m}^{2}$ and $\geq 2$ metabolic risk factors. The MADO subjects were drug-naïve for anti-diabetic medications prior to recruitment. A written informed consent was obtained from the participants prior to their inclusion in the study. The study was carried out according to the Declaration of Helsinki and approved by the Institutional Ethics Committee (IEC). All subjects underwent clinical and biochemical assessment. Detailed anthropometry such as height, weight, BMI, waist circumference (WC), body fat percentage and blood pressure $(\mathrm{mmHg})$ were recorded using the standard methods. The samples were collected after 10-12 h of overnight fasting. Study subjects were screened for glucose intolerance by standard oral glucose tolerance test (OGTT) using $75 \mathrm{~g}$ of anhydrous glucose as per the American Diabetes Association (ADA) criteria [17].

\section{Biochemical investigations}

Various biochemical parameters such as fasting plasma insulin (FPI) by electrochemiluminescence immunoassay and $\mathrm{HbA}_{1 \mathrm{C}}$ (Bio-Rad D10 system, Hercules, CA, USA) was determined by ion-exchange high-performance liquid chromatography (HPLC) method. Plasma glucose and lipid profile were estimated by an enzymatic method (Roche autoanalyser, Mannheim, Germany). The insulin resistance and $\beta$-cell function were calculated using the homeostasis model assessment HOMA-IR and HOMA- $\beta$ by the standard formula [18].

\section{PBMCs isolation}

Around 3-4 $\mathrm{ml}$ of whole blood was collected from all the study subjects. PBMCs were isolated using FicollHypaque (Sigma-Aldrich, St Louis, MO, USA) density gradient centrifugation method and were washed twice with 1X phosphate buffer saline (PBS) [19]. Cells were counted, and viability was assessed by trypan blue exclusion assay (> 95\% PBMCs were viable). PBMCs obtained from the study subjects were used for determining the mitochondrial reactive oxygen species (mtROS) production and mitochondrial membrane potential (MMP), as well as mRNA and protein expression and TEM studies.

\section{Measurement of proinflammatory cytokines}

Levels of IL-6 (BD Biosciences, USA) and highly sensitive $\mathrm{C}$-reactive protein (hsCRP) (Diagnostics Biochem,
Canada) were determined by sandwich enzyme-linked immunosorbent assay (ELISA) in MHNO, MHO and MADO subjects according to the manufacturer's instructions. The samples were read on an ELISA microplate reader (TECAN, Salzburg, Austria) at a test wavelength of $450 \mathrm{~nm}$.

\section{Mitochondrial reactive oxygen species (mtROS) measurement}

Freshly isolated PBMCs were incubated with $5 \mu \mathrm{M}$ MitoSOX $^{\mathrm{TM}}$ Red reagent (Molecular Probes, Invitrogen, $\mathrm{CA}$, USA) for $10 \mathrm{~min}$ at $37{ }^{\circ} \mathrm{C}$ in a humidified $5 \% \mathrm{CO}_{2}$ incubator, under dark conditions. After the completion of the incubation time, the cells were washed twice with $1 \mathrm{X}$ PBS and subjected to flow cytometry (Becton Dickinson FACS Aria II) and the data was analyzed using BD FACSDiva software (Becton Dickinson, Franklin Lakes, NJ, USA).

\section{Detection of mitochondrial membrane potential (MMP)}

Briefly for JC-1 staining, PBMCs were incubated with JC-1 stain (1:100 dilution in $1 \times$ JC-1 assay buffer) (BD Biosciences, USA) for $10 \mathrm{~min}$, suspended in $1 \times \mathrm{JC}-1$ assay buffer at $37{ }^{\circ} \mathrm{C}$ with $5 \% \mathrm{CO}_{2}$. The cells were washed twice with $1 \mathrm{X}$ PBS and subsequently resuspended in $1 \times$ assay buffer for flow cytometric analysis. The entire protocol was performed under dark conditions. Data were acquired using the BD FACS Aria II flow cytometer (Becton Dickinson Bio-sciences) and analyzed by BD FACSDiva software version 6.1.2 (Becton Dickinson, Franklin Lakes, NJ, USA).

\section{RNA isolation and real-time quantitative PCR (qRT-PCR)}

Total cellular RNA was extracted from PBMCs by TRIZOL reagent (Life Technologies) and $2 \mu \mathrm{g}$ of RNA was reverse transcribed to cDNA template by reversetranscriptase PCR. Real-time PCR amplifications of mitophagy-related genes, i.e. PINK1, PARKIN, NIX, MFN2, LC3-II and LAMP-2 were performed in duplicates in $20 \mu \mathrm{l}$ reaction volume by SYBR green chemistry (Applied Biosystems). Human-specific primers were used (Applied Biosystems). The data is represented as relative mRNA expression normalized to human $\beta$-actin mRNA expression. The quantification of gene expression was calculated by $2^{-\Delta \mathrm{CT}}$ method, which is expressed in fold change [20]. The relative fold change in the MHO and MADO groups was normalized with the fold change of MHNO subjects.

\section{Western blotting}

PBMCs were lysed in RIPA buffer $(50 \mathrm{mM}$ Tris- $\mathrm{HCl}$ $\mathrm{pH}$ 7.6, $150 \mathrm{mM} \mathrm{NaCl}, 1 \mathrm{mM}$ EGTA, 1\% Triton NP-40, $1 \%$ sodium deoxycholate, $0.1 \%$ SDS, $50 \mathrm{mM} \mathrm{NaF}$ ), 
supplemented with protease inhibitors (Sigma-Aldrich, USA). Cell lysates were centrifuged at 10,000 rpm and the protein concentration was determined in the supernatant samples by Qubit reagent (Molecular Probes, Invitrogen, CA, USA). $70 \mu \mathrm{g}$ of protein was resolved on $12.5 \%$ SDS-polyacrylamide gels and transferred onto PVDF membranes (Millipore, Bedford, MA) using a semi-dry transfer system (Amersham Biosciences, GE Healthcare, USA). PVDF membranes were blocked with $5 \%$ skimmed milk in Tris-buffered saline Tween buffer (TBST; $20 \mathrm{mM}$ Tris, $137 \mathrm{mM} \mathrm{NaCl}$, pH 7.6, with 0.1\% TWEEN 20) overnight at $4{ }^{\circ} \mathrm{C}$. The blots were incubated with primary antibodies against PINK1 $(1 \mu \mathrm{g} / \mathrm{ml}$; Sigma-Aldrich, USA), PARKIN $(1 \mu \mathrm{g} / \mathrm{ml})$, MFN2 $(2.51 \mu \mathrm{g} / \mathrm{ml})$, NIX $(0.75 \mu \mathrm{g} /$ $\mathrm{ml})$, LC3-II $(1.5 \mu \mathrm{g} / \mathrm{ml})$ and LAMP-2 $(2 \mu \mathrm{g} / \mathrm{ml})$, obtained from Abcam (Cambridge, MA, USA), in TBST for $2 \mathrm{~h}$ at room temperature. After three washings with TBST, the membrane was probed with their respective HRP-conjugated secondary antibodies for $1 \mathrm{~h}$ at room temperature. Immunoblot images were detected using an ECL substrate (Thermo Scientific, Waltham, MA, USA) by an enhanced chemiluminescence system (Fluorchem M, Protein simple). The band intensity of target proteins was measured by Image J software (1.47 v) and normalized to levels of $\beta$-actin, which was used as a loading control.

\section{Transmission electron microscopy (TEM)}

For TEM studies, the PBMCs collected from the study subjects were fixed with $3 \%$ glutaraldehyde in $0.2 \mathrm{M}$ Sorensen's phosphate buffer ( $\mathrm{pH}$ 7.4) for $2-4 \mathrm{~h}$, followed by $1 \%$ osmium tetroxide in Millionig's buffer of the same $\mathrm{pH}$ for $2 \mathrm{~h}$. The samples were subsequently dehydrated through an ascending ethanol series (70, 90, 100\%), and embedded in an epon resin followed by polymerization at $60{ }^{\circ} \mathrm{C}$ for $24 \mathrm{~h}$. Ultrathin sections $(50 \mathrm{~nm})$ were obtained using an ultramicrotome and mounted on the EM grids. The sections were stained with $1 \%$ uranyl acetate for 20 min and observed under a JEM 1400 Plus electron microscope (Jeol Ltd., Tokyo, Japan). The total mitochondrial number and percentage of damaged mitochondria per cell were evaluated by two independent examiners in a blinded manner. Image J software was used to assess the morphology of damaged mitochondria, which was defined by aspect ratio (length of the major axis/ minor axis) and form factor (perimeter ${ }^{2} / 4 \pi$ *area), depicting the size and degree of mitochondrial branching, respectively.

\section{Statistical analysis}

Data are expressed as mean \pm SD for the parametric data and median (interquartile range) for the nonparametric variables. One-way analysis of variance (ANOVA) for parametric data, while Kruskal Wallis $\mathrm{H}$ test for non-parametric data was employed to compare the groups, followed by a post hoc test (Bonferroni or Dunn's multiple comparison tests, respectively). Spearman correlation coefficient was calculated to see relationship between different variables. Values with $\mathrm{p}<0.05$ were considered statistically significant. The statistical analysis was performed using the SPSS version 22 software for window (SPSS Inc., Chicago, USA).

\section{Results}

\section{Clinical and metabolic characteristics}

The clinical and biochemical characteristics of the study subjects are summarized in Table 1 . The anthropometric measurements including weight, $\mathrm{BMI}, \mathrm{WC}$, and body fat $\%$ were significantly higher in $\mathrm{MHO}$ and MADO subjects as compared to the MHNO group, while they were comparable between MHO and MADO subjects $(\mathrm{p}<0.05)$. Further, other evidence for markers of insulin resistance like acanthosis nigricans and skin tags were observed to be highly prevalent in the $\mathrm{MHO}(50 \%$ and $15 \%)$ and MADO (85\% and $45 \%$ ) groups as compared to the MHNO subjects (30\% and 5\%), respectively. Systolic blood pressure (SBP) and diastolic blood pressure (SBP) were higher in

Table 1 Clinical and biochemical characteristics of metabolically healthy non-obese (MHNO), metabolically healthy obese (MHO) and metabolically abnormal diabetic obese (MADO) subjects

\begin{tabular}{|c|c|c|c|}
\hline Variables & $\begin{array}{l}\text { MHNO } \\
(n=20)\end{array}$ & MHO $(n=20)$ & MADO $(n=20)$ \\
\hline Age (years) & $38.9 \pm 7.2$ & $41.5 \pm 6.5$ & $43.7 \pm 10.8^{*}$ \\
\hline Gender (M:F) & $12: 8$ & $11: 9$ & $11: 9$ \\
\hline Wt (kg) & $63.8 \pm 7.7$ & $72.2 \pm 8.3^{*}$ & $73.1 \pm 10.5^{*}$ \\
\hline $\mathrm{BMI}\left(\mathrm{kg} / \mathrm{m}^{2}\right)$ & $21.2 \pm 1.6$ & $28.5 \pm 2.4^{* * *}$ & $29.4 \pm 3.5^{* * *}$ \\
\hline WC $(\mathrm{cm})$ & $81.0 \pm 5.2$ & $96.3 \pm 7.7^{* * *}$ & $98.6 \pm 8.0^{* * *}$ \\
\hline Body Fat (\%) & $22.8 \pm 6.2$ & $33.6 \pm 7.4^{*}$ & $35.0 \pm 10.4^{* * *}$ \\
\hline $\begin{array}{l}\text { Systolic BP (mm/ } \\
\quad \mathrm{Hg})\end{array}$ & $119.7 \pm 12.1$ & $122.7 \pm 10.7$ & $126.2 \pm 15.2$ \\
\hline $\begin{array}{l}\text { Diastolic BP (mm/ } \\
\quad \mathrm{Hg})\end{array}$ & $80.7 \pm 7.9$ & $85.0 \pm 6.1$ & $81.8 \pm 9.6$ \\
\hline FPG (mmol/l) & $5.1 \pm 0.4$ & $5.2 \pm 0.7$ & $8.6 \pm 1.4^{* * * \# \# \#}$ \\
\hline $2 \mathrm{hPG}(\mathrm{mmol} / \mathrm{l})$ & $5.6 \pm 1.3$ & $6.8 \pm 1.2$ & $13.6 \pm 2.7^{* * * \# \# \#}$ \\
\hline $\mathrm{HbA}_{1 \mathrm{C}}(\%)$ & $5.2 \pm 0.2$ & $5.3 \pm 0.4$ & $7.7 \pm 0.5^{* * * \# \# \#}$ \\
\hline FPI $(\mu \mathrm{lU} / \mathrm{ml})$ & $9.7 \pm 6.2$ & $11.9 \pm 5.1$ & $13.6 \pm 4.7$ \\
\hline HOMA-IR & $2.2 \pm 1.4$ & $2.4 \pm 1.0$ & $5.4 \pm 2.5^{* * * \# \# \#}$ \\
\hline HOMA- $\beta \%$ & $119.5 \pm 83.6$ & $156.3 \pm 81.1$ & $54.2 \pm 17.6^{* * * \# \# \#}$ \\
\hline S. CHOL (mg/dl) & $185.2 \pm 39.1$ & $180.4 \pm 37.5$ & $206.9 \pm 44.8$ \\
\hline S. LDL-C (mg/dl) & $112.5 \pm 27.9$ & $108.1 \pm 32.2$ & $141.0 \pm 36.7^{* \#}$ \\
\hline S. TG (mg/dl) & $134.2 \pm 77.8$ & $140.4 \pm 70.2$ & $152.2 \pm 57.3$ \\
\hline S. HDL-C (mg/dl) & $44.2 \pm 7.7$ & $50.1 \pm 16.7$ & $44.2 \pm 6.7$ \\
\hline IL-6 (pg/ml) & $6.9 \pm 1.1$ & $8.2 \pm 2.2$ & $15.2 \pm 2.6^{* * \#}$ \\
\hline hsCRP (mg/dl) & $0.7 \pm 0.4$ & $1.7 \pm 1.2^{*}$ & $3.8 \pm 2.3^{* * * \#}$ \\
\hline
\end{tabular}

Data expressed as mean $\pm S D,{ }^{*} p<0.05,{ }^{* *} p<0.01,{ }^{* * *} p<0.001$ vs. MHNO \# $\mathrm{p}<0.05,{ }^{\# \#} \mathrm{p}<0.01, \# \#$ $\mathrm{p}<0.001$ vs. MHO 
the $\mathrm{MHO}$ and MADO groups, though non-significant. All subjects in MHNO and MHO group were normotensive, while $15 \%$ of MADO patients received anti-hypertensive drugs. The biochemical parameters including FPG, 2hPG and $\mathrm{HbA}_{1 \mathrm{C}}$ levels were significantly higher in the MADO group as compared to their $\mathrm{MHO}$ and $\mathrm{MHNO}$ counterparts $(\mathrm{p}<0.05)$. The lipid and lipoprotein profile was comparable between the MHNO and MHO subjects, while the MADO subjects displayed higher levels, though nonsignificant, except for LDL-C levels, which were significantly higher as compared to the other groups $(\mathrm{p}<0.05)$. Interestingly, the MHO subjects displayed a preserved insulin sensitivity as reflected by the comparable HOMAIR indices relative to the MHNO participants. However, the MADO subjects displayed higher levels of HOMA-IR than the other groups $(p<0.05)$. Further, HOMA- $\beta$ indices were significantly reduced in MADO group as compared to the MHO and MHNO subjects $(\mathrm{p}<0.05)$.

\section{Levels of proinflammatory cytokines}

MHO and MADO groups exhibited significantly higher serum levels of hsCRP relative to the MHNO subjects $(\mathrm{p}<0.05)$. IL-6 levels were higher, though insignificant in MHO subjects as compared to the MHNO group, while they were significantly higher in the MADO group as compared to the other groups $(\mathrm{p}<0.05)$ (Table 1$)$.

\section{mtROS content and MMP}

As compared to the MHNO group, the MHO subjects exhibited higher mitochondrial oxidative stress levels as evidenced by significantly increased mtROS production $(\mathrm{p}<0.05)$. However, MMP, which is expressed as the percentage of cells with collapsed membrane potential, showed a moderate increase in the MHO subjects, though it was insignificant. Further, the mtROS content and collapsed MMP were significantly higher the in MADO subjects ( $p<0.05$ vs. both MHNO and MHO groups) (Fig. 1a-d).

\section{Correlation between body composition, $\mathrm{HbA}_{1 \mathrm{C}}$ and mitochondrial ROS content}

A significant and positive correlation of BMI $(r=0.440$; $\mathrm{p}<0.05)$ and body fat percentage $(\mathrm{r}=0.518 ; \mathrm{p}<0.01)$ with the increased mitochondrial ROS content was observed in the MHO participants. Similar correlation was also noted with BMI $(\mathrm{r}=0.794 ; \mathrm{p}<0.001)$ and body fat percentage $(r=0.686 ; p<0.001)$ in the MADO patients. Though the severity of mitochondrial oxidative stress was more, as MOS also showed a positive correlation with the rising $\mathrm{HbA}_{1 \mathrm{C}}$ levels, reflected by $\mathrm{MMP}$ $(\mathrm{r}=0.780 ; \mathrm{p}<0.01)$ and $\mathrm{mtROS}(\mathrm{r}=0.812 ; \mathrm{p}<0.01)$ in these patients (Fig. 2a-f).
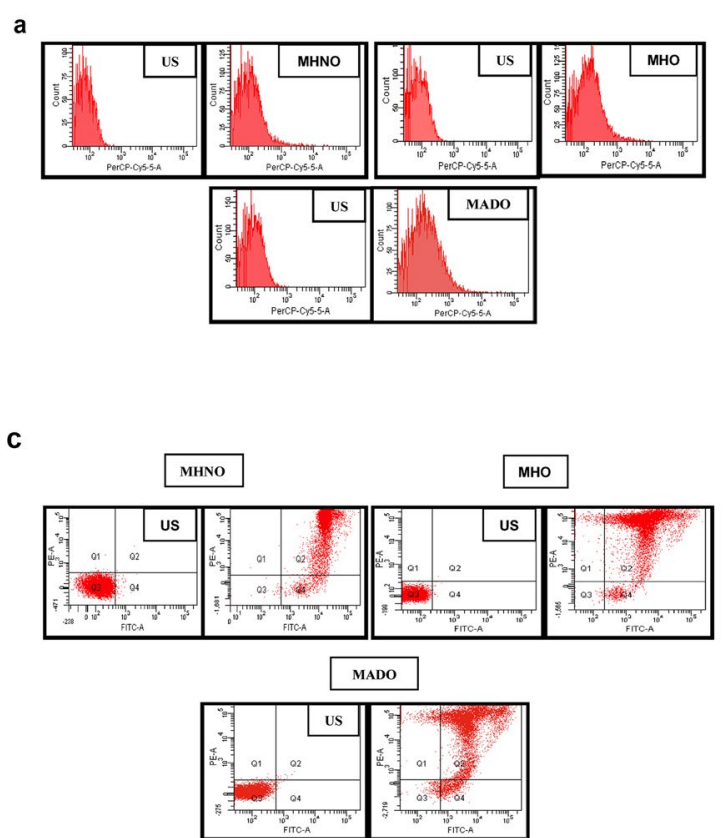

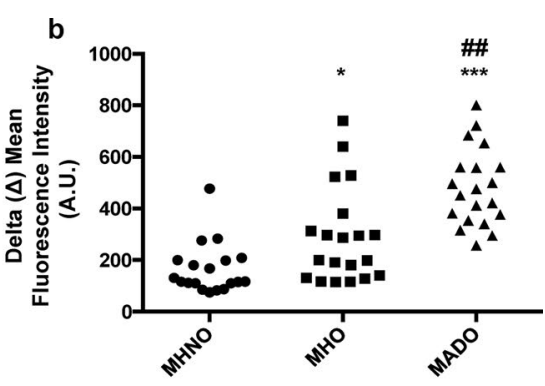

d

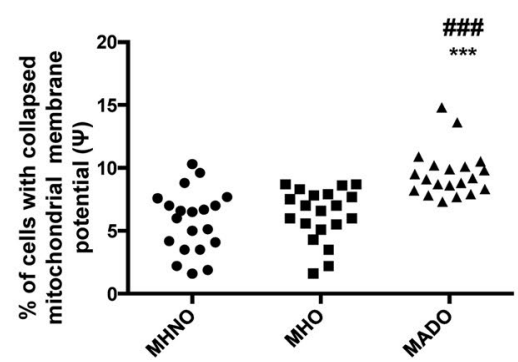

Fig. 1 Depicts mtROS content and MMP in the study subjects. a Representative FACS image of Mitosox fluorescence intensity. b Bar graph represents delta $(\triangle)$ mean fluorescence intensity of Mitosox in metabolically healthy non-obese (MHNO), metabolically healthy obese $(\mathrm{MHO})$ participants and $(n=20$ each) and metabolically abnormal diabetic obese (MADO) participants. c Representative scatter plot of MMP. $\mathbf{d}$ Bar diagram showing the percentage of cells with collapsed MMP in MHNO, MHO and MADO subjects. Values are expressed in median and interquartile range $(n=20$ each), $\left(^{*}=\right.$ vs. MHNO), $\left({ }^{\#}=\right.$ vs. MHO), ${ }^{*} p<0.05,{ }^{* * *} p<0.001,{ }^{\# \#} p<0.01,{ }^{\# \#} p<0.001$ 

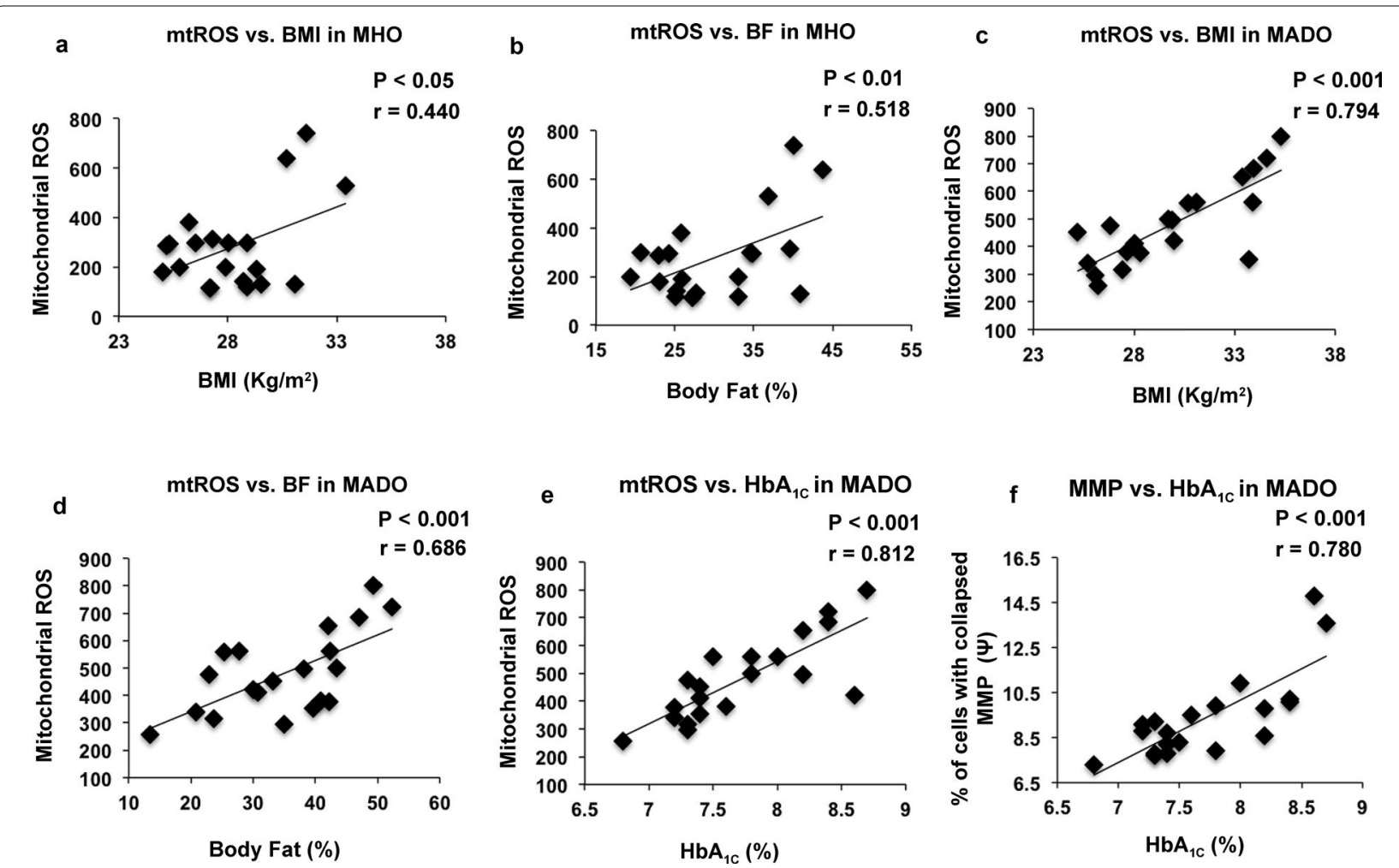

Fig. 2 Scatter plot illustrating the correlation of mitochondrial ROS ( $m$ tROS) vs. BMI and body fat in the MHO group (a, b), BMI and body fat in the MADO group (c, d). Correlation graph displaying the relationship between the increasing $\mathrm{HbA}_{1 \mathrm{C}}$ levels and mtROS content, and MMP in the MADO subjects $(\mathbf{e}, \mathbf{f})$

Transcriptional profiling of mitophagy-related genes mRNA expression of PINK1 gene was significantly attenuated in the MHO subjects as compared to the MHNO group ( $\mathrm{p}<0.05)$. However, no significant alterations in the mRNA expression of other mitophagy-related markers including MFN2, PARKIN, NIX, LC3-II and LAMP-2 genes was observed between both the $\mathrm{MHO}$ and $\mathrm{MHNO}$ subjects, whereas, a significant downregulation in the mRNA levels of mitophagy-related genes was observed in the MADO patients with respect to $\mathrm{MHNO}$ and $\mathrm{MHO}$ groups $(\mathrm{p}<0.05)$ (Fig. 3a-f).

\section{Translational profiling of mitophagy-markers}

Western blot analysis revealed that there was no significant difference in the protein expression of PARKIN, MFN2, NIX, LC3-II and LAMP-2 except for PINK1 protein, which was significantly downregulated in the $\mathrm{MHO}$ subjects as compared to the MHNO group ( $<<0.05$ ). Moreover, MADO group displayed a significantly attenuated expression profile of mitophagy-related proteins as compared to the other groups ( $<$ 0.05) (Fig. 4a-g).

\section{Quantification of damaged mitochondria}

TEM analysis demonstrated that there was no significant difference in the total mitochondrial number per cell among all the study subjects. However, a significant increase in the percentage of damaged mitochondria was observed in the MADO patients relative to the $\mathrm{MHO}$ and $\mathrm{MHNO}$ groups, as depicted by significantly reduced aspect ratio and form factor $(\mathrm{p}<0.05)$. However, subjects in $\mathrm{MHO}$ and $\mathrm{MHNO}$ groups displayed almost similar percentage of damaged mitochondria (Fig. 5a-d; Table 2).

\section{Correlation between the damaged mitochondria and mitophagy}

Correlation analysis revealed that the percentage of damaged mitochondria showed a significant and negative correlation with the mRNA levels of mitophagy-related markers including NIX $(\mathrm{r}=-0.702 ; \mathrm{p}=0.035)$, PARKIN $(\mathrm{r}=-0.680 ; \mathrm{p}=0.044)$, LC3-II $(\mathrm{r}=-0.761, \mathrm{p}=0.017)$ and LAMP-2 genes $(\mathrm{r}=-0.707 ; \mathrm{p}=0.033)$.

\section{Discussion}

The present study demonstrates that obesity is associated with an increased mitochondrial oxidative stress, but did not significantly influence mitophagy in $\mathrm{MHO}$ subjects. However, the MADO patients with similar body composition as MHO group were accompanied by metabolic abnormalities, increased levels of mitochondrial oxidative stress, and displayed attenuated mitophagy. Our 

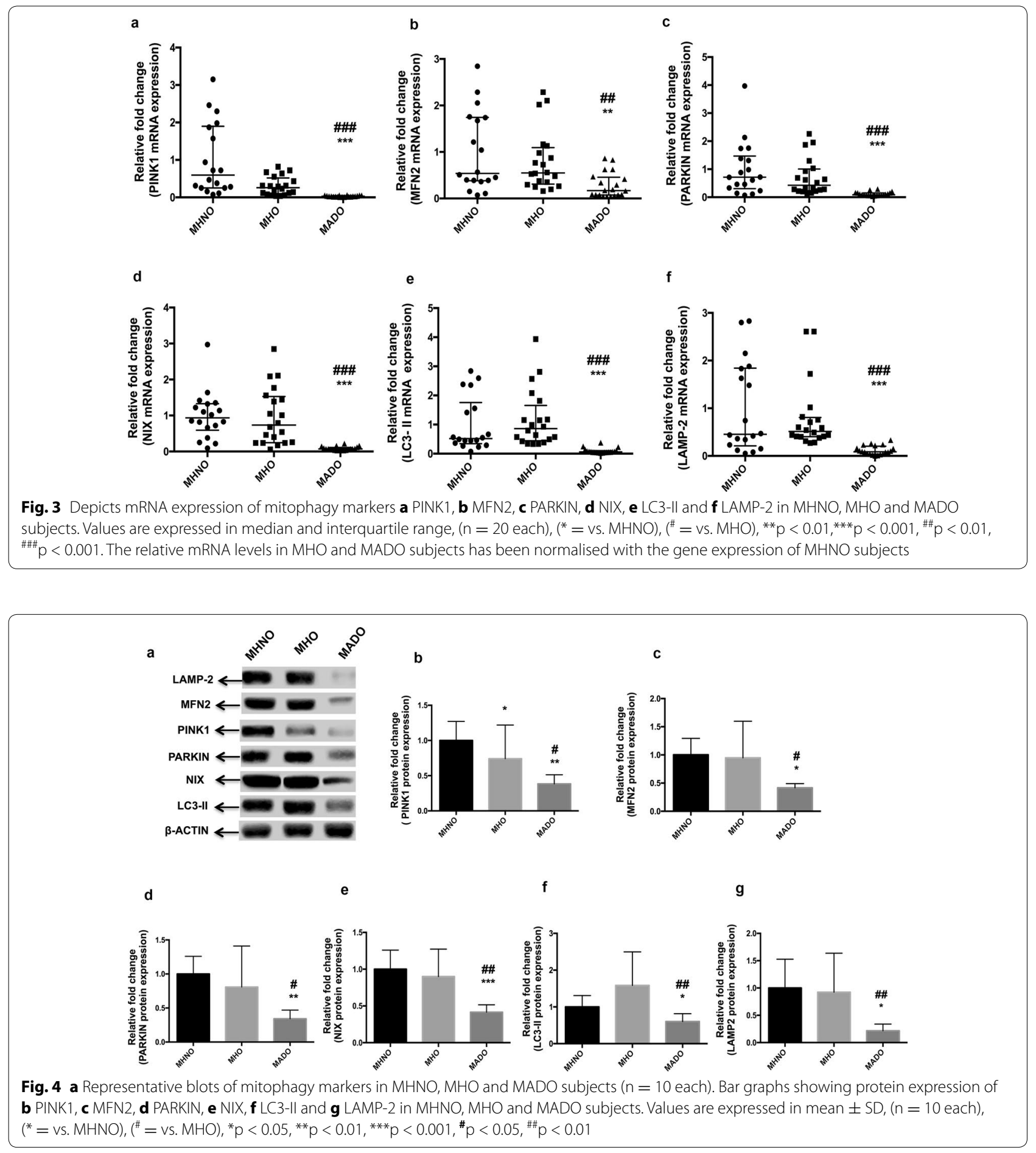

observations indicate that obesity-mediated oxidative stress doesn't significantly alter mitophagy, unless accompanied by metabolic abberations as seen in the MADO patients. Therefore, it is conceivable that the favorable metabolic profile contributes to sustenance of mitophagy in response to mild oxidative stress levels in the $\mathrm{MHO}$ group and this may further limit the rising mitochondrial oxidative stress in these subjects.

Obesity-mediated oxidative stress has been linked to metabolic diseases like diabetes and atherosclerosis, as increased adiposity in the obese individuals results in an increased oxidative stress and mitochondrial dysfunction, 


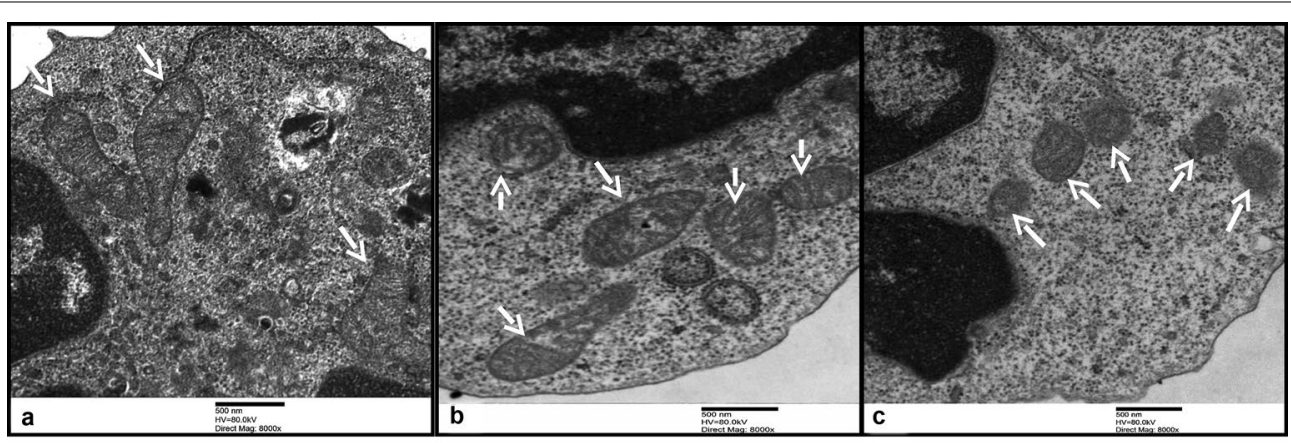

d

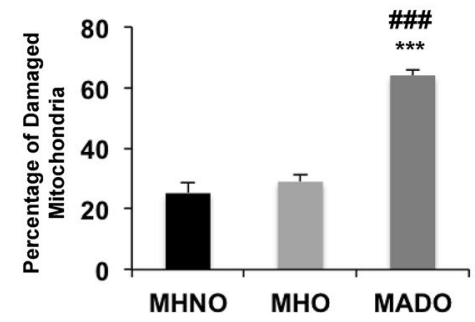

Fig. 5 Electron micrographs of PBMCs showing mitochondria in a MHNO, b MHO and $\mathbf{c} M A D O$ groups. Mitochondria are represented by white arrows. Magnification: ×8000, Scale bar: $500 \mathrm{~nm}$. MHNO and MHO groups displayed healthy mitochondria, which are larger in size, elliptical/oval in shape with well-preserved cristae, while MADO patients showed smaller and fragmented mitochondria. $\mathbf{d}$ Bar diagram displaying the percentage of damaged mitochondria in the respective study groups. Data expressed as mean $\pm \mathrm{SD}\left(\mathrm{n}=3\right.$ each), $\left({ }^{*}=\mathrm{vs}\right.$. MHNO), $\left({ }^{*}=\mathrm{vs} . \mathrm{MHO}\right),{ }^{* * *} \mathrm{p}<0.001$, $\# \#<0.01)$

Table 2 Mitochondrial morphological characteristics in metabolically MHNO, MHO and MADO subjects

\begin{tabular}{lrrr}
\hline $\begin{array}{l}\text { Morphological char- } \\
\text { acteristics }\end{array}$ & MHNO $(\mathbf{n}=\mathbf{3})$ & MHO $(\mathbf{n}=\mathbf{3})$ & MADO $(\mathbf{n}=\mathbf{3})$ \\
\hline Mitochondria (\#/cell) & $6.8 \pm 1.5$ & $7.2 \pm 1.3$ & $7.1 \pm 0.5$ \\
Aspect ratio & $1.81 \pm 0.5$ & $1.84 \pm 0.9$ & $1.20 \pm 0.4^{* * \#}$ \\
$\begin{array}{l}\text { Form factor } \\
\text { \% of damaged mito- } \\
\text { chondria }\end{array}$ & $25.63 \pm 0.1$ & $1.62 \pm 0.5$ & $1.16 \pm 0.4^{* \# \#}$ \\
\hline
\end{tabular}

Data expressed as mean $\pm S D$, ${ }^{* *} \mathrm{p}<0.01,{ }^{* * *} \mathrm{p}<0.001$ vs. MHNO

$\# \mathrm{p}<0.05, \# \mathrm{p}<0.01, \# \mathrm{p}<0.001$ vs. MHO

an underlying cause of insulin resistance [21, 22]. Our data revealed that the MHO subjects exhibited increased mitochondrial ROS content, which showed a positive and significant correlation with BMI and body fat percentage. However, a non-significant increase in the collapsed MMP in MHO subjects could be attributed to the sustained mitophagic process, resulting in the engulfment of the damaged and defunct mitochondria. This phenomenon may result in limiting the aggravated mitochondrial oxidative stress levels, as these defunct mitochondria are the potential source of ROS generation.
These observations indicate that the MHO subjects display a higher degree of mitochondrial oxidative stress as compared to the MHNO subjects due to the higher BMI and body fat percentage. However, MHO subjects have been shown to display moderately lower levels of oxidative stress as compared to the MADO individuals, with similar BMI and body fat, though accompanied with altered metabolic profile. In this context, Bañuls et al. also reported that the mitochondrial ROS production was enhanced in MADO subjects as compared to the MHO group [23]. Our study revealed that MADO patients exhibited even higher mtROS content and collapsed MMP as compared to the MHNO and MHO groups. Further, mitochondrial stress indices also showed a positive correlation with body composition and rising $\mathrm{HbA}_{1 \mathrm{c}}$ levels, indicating that the adiposity accompanied with glucotoxicity may have contributed to an aggravated mitochondrial oxidative stress in these patients.

Reports in the literature demonstrate that the MHO individuals may not be predisposed to the similar risk of developing metabolic abnormalities as compared to the MADO subjects. Nonetheless, the underlying mechanisms for the apparently healthy metabolic profile of the MHO subjects remain conjectural. Accumulating evidence reports that the preferential autophagic processes 
like mitophagy are triggered in response to ROS [24]. To the best of our knowledge, this is the first report in the literature to assess the status of mitophagy in metabolically healthy obese (MHO) subjects, particularly in Asian Indians. Our observations revealed that the mRNA and protein expression of mitophagy markers in MHO subjects remained unaltered and akin to that of the MHNO subjects, except for PINK1, which was significantly downregulated in $\mathrm{MHO}$ group. However, in patients with MADO, the expression of mitophagy-related markers was significantly attenuated as compared to MHO and MHNO groups. Sustenance of mitophagy in MHO participants can be explained by the fact that the magnitude of mitochondrial oxidative stress was moderate, and may not have attained a particular threshold level that could have significantly altered the mitophagy. Moreover, the favourable metabolic phenotype in these MHO subjects may also have contributed to the the sustenance of mitophagy by limiting ROS generation. Nevertheless, attenuated PINK1 expression in MHO subjects may be explained by the fact that PINK1 is highly susceptible to oxidative stress-induced proteolysis, thereby, rendering it non-functional [25]. Interestingly, the HOMA-IR levels were not significantly different in MHO subjects as compared to the MHNO subjects, indicating a crucial role of mitophagy in maintaining mitochondrial health and consequent insulin sensitivity. As far as MADO patients are concerned, multiple metabolic abnormalities and higher levels of mitochondrial oxidative stress may result in oxidative damage of mitochondrial proteins, which generates "mitophagy-resistant" mitochondria, leading to impaired mitophagy and consequent accumulation of dysfunctional mitochondria and increased ROS generation. Our findings indicate that phenotypic alterations are not suffice to exert significant impact on mitophagy unless accompanied by severe metabolic abnormalities as observed in patients with MADO.

Further, TEM analysis demonstrated an increased number of damaged mitochondria in patients with MADO and also showed a negative correlation with the mitophagy-related markers, indicating that metabolic abnormalities-induced mitochondrial oxidative stress led to attenuated mitophagy, resulting in the accumulation of dysfunctional mitochondria. Of note, the alterations in mitochondrial oxidative stress and mitophagy are of particular importance in PBMCs of MHO and MADO subjects, as these cells have been shown to reflect oxidative stress and inflammation associated with obesity $[26,27]$.

Obesity is also a state of chronic inflammation and contributes as a major risk factor for the development of T2DM. Therefore, there is a likelihood that inflammasome regulation plays an important role in the induction and pathogenesis of the inflammatory disorders like obesity and diabetes [28]. Moreover, a recent report by Kim et al. revealed that mitophagy negatively regulates inflammasome activation by engulfing the damaged mitochondria, as these superfluous mitochondria act as potential activators of inflammasomes [29]. Though we did not assess the inflammasome activity in the study subjects; our data demonstrated that sustained mitophagy in MHO subjects may inhibit inflammasome activation, which may further reduce inflammation in these subjects, despite having higher BMI. Thus, our observations indicate that mitophagy, a cell-reparative process, is a self-limiting phenomenon that may protect the cells from excessive inflammation, contributing to a low-grade inflammatory profile in MHO subjects.

In conclusion, our study highlights the importance of favourable metabolic profile in MHO subjects, which may be one of the contributing factor in the sustenance of mitophagic process, a beneficial metabolic event. This phenomenon results in reduced mitochondrial oxidative stress, low-grade inflammation, thereby, leading to improved mitochondrial function and preserved insulin sensitivity. Thus, these findings strengthen the understanding of pathophysiological mechanisms underlying the reduced risk of developing metabolic abnormalities due to lower oxidative stress and inflammatory profile in the MHO subjects.

\begin{abstract}
Abbreviations
MHNO: metabolically healthy non-obese; $\mathrm{MHO}$ : metabolically healthy obese; MADO: metabolically abnormal diabetic obese; T2DM: type 2 diabetes mellitus; ROS: reactive oxygen species; HOMA-IR: homeostatic model assessment of insulin resistance; HOMA- $\beta$ : homeostatic model assessment of $\beta$-cell function; mtROS: mitochondrial reactive oxygen species; MMP: mitochondrial membrane potential.
\end{abstract}

\section{Authors' contributions}

Study patients: $A B$, data analysis and interpretation: $S B, A B$ and VD. Manuscript writing: $S B$, manuscript editing: $A B$ and $V D$. All authors read and approved the final manuscript.

\section{Author details}

${ }^{1}$ Department of Experimental Medicine and Biotechnology, Postgraduate Institute of Medical Education and Research (PGIMER), Research Block-B, Chandigarh 160012, India. ${ }^{2}$ Department of Endocrinology, Postgraduate Institute of Medical Education and Research (PGIMER), Chandigarh, India.

\section{Acknowledgements}

We greatly acknowledge the kind help of Prof. Uma Nahar Saikia and Dr. CS Rayat for accomplishment of TEM studies. We would also like to thank Dr. Rama Walia for her inputs to the clinical study, Mr. Vivek Sharma for technical assistance, Dr. Shobhit Bhansali for statistical analysis and all the study participants for their cooperation during the study period.

\section{Competing interests}

The authors declare that they have no competing interests.

\section{Availability of data and materials}

The datasets supporting the conclusion of this work are included in the article and its additional files. 


\section{Consent for publication}

The authors confirm that they have obtained the consent from the study participants to publish the data.

\section{Ethical approval and consent to participate}

The study was performed according to the Declaration of Helsinki and was approved by the Institutional Ethics Committee (IEC) at Post Graduate Institute of Medical Education and Research (PGIMER), Chandigarh. INDIA. An informed consent was obtained from the patients prior to their participation in the study.

\section{Funding}

The financial grant for the study was received from the Department of Biotechnology (DBT), New Delhi, India.

\section{Publisher's Note}

Springer Nature remains neutral with regard to jurisdictional claims in published maps and institutional affiliations.

Received: 6 September 2017 Accepted: 4 December 2017 Published online: 12 December 2017

\section{References}

1. Gómez-Ambrosi J, Silva C, Galofré JC, Escalada J, Santos S, Millán D, et al. Body mass index classification misses subjects with increased cardiometabolic risk factors related to elevated adiposity. Int J Obes (Lond). 2012;36:286-94.

2. Tchernof A, Després JP. Pathophysiology of human visceral obesity: an update. Physiol Rev. 2013:93:359-404.

3. Jung $\mathrm{CH}$, Lee WJ, Song KH. Metabolically healthy obesity: a friend or foe? Korean J Intern Med. 2017:32:611-21.

4. Zheng R, Yang M, Bao Y, Li H, Shan Z, Zhang B, et al. Prevalence and determinants of metabolic health in subjects with obesity in Chinese Population. Int J Environ Res Public Health. 2015;12:13662-77.

5. Geetha L, Deepa M, Anjana RM, Mohan V. Prevalence and clinical profile of metabolic obesity and phenotypic obesity in Asian Indians. J Diabetes Sci Technol. 2011;5:439-46.

6. Mitchell T, Darley-Usmar V. Metabolic syndrome and mitochondrial dysfunction: insights from preclinical studies with a mitochondrially targeted antioxidant. Free Radic Biol Med. 2012:52:838-40.

7. Cerf ME. Beta cell dysfunction and insulin resistance. Front Endocrino (Lausanne). 2013:4:37.

8. Chattopadhyay M, Khemka VK, Chatterjee G, Ganguly A, Mukhopadhyay S, Chakrabarti S. Enhanced ROS production and oxidative damage in subcutaneous white adipose tissue mitochondria in obese and type 2 diabetes subjects. Mol Cell Biochem. 2015:399:95-103.

9. Befroy DE, Petersen KF, Dufour S, Mason GF, de Graaf RA, Rothman DL, et al. Impaired mitochondrial substrate oxidation in muscle of insulinresistant offspring of type 2 diabetic patients. Diabetes. 2007;56:1376-81.

10. Hirota Y, Kang D, Kanki T. The physiological role of mitophagy: new insights into phosphorylation events. Int J Cell Biol. 2012;2012:354914.

11. Twig G, Elorza A, Molina AJ, Mohamed H, Wikstrom JD, Walzer G, et al. Fission and selective fusion govern mitochondrial segregation and elimination by autophagy. EMBO J. 2008;27:433-46.

12. Ding WX, Yin XM. Mitophagy: mechanisms, pathophysiological roles, and analysis. Biol Chem. 2012;393:547-64.

13. Bournat JC, Brown CW. Mitochondrial dysfunction in obesity. Curr Opin Endocrinol Diabetes Obes. 2010;17:446-52.

14. Hu F, Liu F. Mitochondrial stress: a bridge between mitochondrial dysfunction and metabolic diseases? Cell Signal. 2011;23:1528-33.
15. Consultation WHOE. Appropriate body-mass index for Asian populations and its implications for policy and intervention strategies. Lancet. 2004;363:157-63.

16. Chackrewarthy S, Gunasekera D, Pathmeswaren A, Wijekoon CN, Ranawaka UK, Kato N, Takeuchi F, Wickremasinghe AR. A comparison between revised NCEP ATP III and IDF definitions in diagnosing metabolic syndrome in an Urban Sri Lankan population: the ragama health study. ISRN Endocrinol. 2013;2013:320176

17. American Diabetes Association. Diagnosis and classification of diabetes mellitus. Diabetes Care. 2014;37:S81-90.

18. Matthews DR, Hosker JP, Rudenski AS, Naylor BA, Treacher DF, Turner RC. Homeostasis model assessment: insulin resistance and beta-cell function from fasting plasma glucose and insulin concentrations in man. Diabetologia. 1985;28:412-9.

19. Boyum A. Separation of leucocytes from blood and bone marrow. Scand J Clin Lab Invest. 1968:21:77.

20. Schmittgen TD, Livak KJ. Analyzing real-time PCR data by the comparative C (T) method. Nat Protoc. 2008:3:1101-8.

21. Yubero-Serrano EM, Delgado-Lista J, Peña-Orihuela P, Perez-Martinez P, Fuentes F, Marin C, et al. Oxidative stress is associated with the number of components of metabolic syndrome: LIPGENE study. Exp Mol Med. 2013;45:e28.

22. Houstis N, Rosen ED, Lander ES. Reactive oxygen species have a causal role in multiple forms of insulin resistance. Nature. 2006;440:944-8.

23. Bañuls C, Rovira-Llopis S, Lopez-Domenech S, Diaz-Morales N, Blas-Garcia A, Veses $\mathrm{S}$, et al. Oxidative and endoplasmic reticulum stress is impaired in leukocytes from metabolically unhealthy vs healthy obese individuals. Int J Obes. 2017:41(10):1556-63 (Epub ahead of print)

24. Scherz-Shouval R, Elazar Z. ROS, mitochondria and the regulation of autophagy. Trends Cell Biol. 2007;17:422-7

25. Fang EF, Scheibye-Knudsen M, Brace LE, Kassahun H, SenGupta T, Nilsen H, Mitchell JR, Croteau DL, Bohr VA. Defective mitophagy in XPA via PARP-1 hyperactivation and NAD(+)/SIRT1 reduction. Cell. 2014;157:882-96.

26. Catalán V, Gómez-Ambrosi J, Rodríguez A, Ramírez B, Valentí V, Moncada $\mathrm{R}$, et al. Peripheral mononuclear blood cells contribute to the obesityassociated inflammatory state independently of glycemic status: involvement of the novel proinflammatory adipokines chemerin, chitinase-3-like protein 1, lipocalin-2 and osteopontin. Genes Nutr. 2015;10:460.

27. Monzo-Beltran L, Vazquez-Tarragón A, Cerdà C, Garcia-Perez P, Iradi A, Sánchez C, et al. One-year follow-up of clinical, metabolic and oxidative stress profile of morbid obese patients after laparoscopic sleeve gastrectomy. 8-oxo-dG as a clinical marker. Redox Biol. 2017;12:389-402.

28. Lukens JR, Dixit VD, Kanneganti TD. Inflammasome activation in obesity-related inflammatory diseases and autoimmunity. Discov Med. 2011:12:65-74

29. Kim MJ, Yoon JH, Ryu JH. Mitophagy: a balance regulator of NLRP3 inflammasome activation. BMB Rep. 2016:49:529-35.

\section{Submit your next manuscript to BioMed Central and we will help you at every step:}

- We accept pre-submission inquiries

- Our selector tool helps you to find the most relevant journal

- We provide round the clock customer support

- Convenient online submission

- Thorough peer review

- Inclusion in PubMed and all major indexing services

- Maximum visibility for your research

Submit your manuscript at www.biomedcentral com/submit 